\title{
Understanding interface effects in perovskite thin films
}

\author{
Marie-Bernadette Lepetit, Bernard Mercey, and Charles Simon \\ CRISMAT, ENSICAEN-CNRS UMR6508, 6 bd. Maréchal Juin, 14050 Caen, FRANCE
}

(Dated: June 26, 2018)

\begin{abstract}
The control of matter properties (transport, magnetic, dielectric,...) using synthesis as thin films is strongly hindered by the lack of reliable theories, able to guide the design of new systems, through the understanding of the interface effects and of the way the substrate constraints are imposed to the material. The present paper analyses the energetic contributions at the interfaces, and proposes a model describing the microscopic mechanisms governing the interactions at an epitaxial interface between a manganite and another transition metal oxide in perovskite structure (as for instance $\mathrm{SrTiO}_{3}$ ). The model is checked against experimental results and literature analysis.
\end{abstract}

The technological importance of spin valves or spin injectors as potential applications of manganese oxides induced a large number of works on manganite thin films 2,3 . For this reason the lost of magnetization of $\mathrm{La}_{2 / 3} \mathrm{Sr}_{1 / 3} \mathrm{MnO}_{3}$ (LSMO) or $\mathrm{La}_{2 / 3} \mathrm{Ca}_{1 / 3} \mathrm{MnO}_{3}$ (LCMO) near an $\mathrm{SrTiO}_{3}$ (STO) interface has been the subject of many interpretations. Let us cite (i) homogeneous substrate strain ${ }^{4}$ (ii) electronic and/or chemical phase separation 5 related to structural inhomogeneities at the interface ${ }^{6}$, (iii) manganese $e_{g}$ orbital reconstruction inducing C-type antiferromagnetism $\frac{7,8}{2}$. None of these interpretations however provide a good understanding of the observed phenomena. For instance, it was shown that an homogeneous substrate strain of the in-plane parameters does not relax for film thickness smaller than $1000 \AA^{5}$, while a drastic change in the transport properties is observed for films thinner than a few unit cells $(\sim 3-4$ on STO substrate $\underset{7.9}{7}, \sim 30$ on $\mathrm{LaAlO}_{3}$ substrate $\left.{ }^{\underline{7}}, \ldots\right)$. In the second hypothesis (ii), there is no clear proposition of the nature of the inhomogeneities, their origin, the way they may act in order to induce the observed properties. Finally, ferromagnetic hysteresis loops were found in very thin films up to only three unit cells $s^{9}$ (u.c.), in contradiction with the proposed C-type AFM ordering resulting from orbital ordering (iii). In any case, whatever the reasons put forward, the existence of a so-called "dead layer" at the interface between the manganite film and most perovskite substrates seems to be established ${ }^{9,10}$. This "dead layer" is of a few unit cells width and exhibits a large decrease of the conductivity ; however its origin is not at all understood.

We believe that a careful analysis allow us to infer a model for the interface effects between a manganite and an oxide substrate with a perovskite structure. The main concepts of our model can be summarized as an energy balance at the interface.

- It is well known that the strongest effect of the substrate is to constrain the film inplane cell parameters to fit the substrate ones.

$$
a_{\text {film }}=a_{\text {substrate }} \quad b_{\text {film }}=b_{\text {substrate }}
$$

This constraint is quite strong since it is associated with bond elongation, i.e. the most energetic vibrational modes 11 . It thus relaxes slowly (not before 250 u.c., $1000 \AA$, on a STO substrate $\left.{ }^{\frac{5}{2}}\right)$. In the literature, it is associated with an u.c. volume constraint $V_{\text {film }} \simeq V_{\text {bulk }}$. While there is indeed, in the film free energy, an elastic term favoring $V_{\text {film }}=V_{\text {bulk }}: \frac{V}{2 \kappa}\left(\frac{\Delta V}{V}\right)^{2}$, this term cannot not be treated as a constraint imposed by the substrate. It should rather be evaluated against the other terms of the constrained film free energy.

- The substrate imposes to the film its in-plane symmetry operations. This constraint is usually weak since, acting on bond angles and dihedral angles, it is related to low energy vibrational modes. One can thus expects that, after constraining the few first cells at the interface, these constraints start to relax according to the energy of the associated vibrational mode.

- The electronic structure of the film and substrate interact at the interface. In particular the possible delocalization effects at the interface should be taken into account.

As a matter of example let us see how these constraints apply to a LSMO thin film on an STO (001) substrate. The in-plane parameters of the LSMO are imposed by the STO substrate and the film in under tensile strain at the interface. As already mentioned, this constraint holds over a large number of monolayers (ML).The STO in-plane symmetry operations impose to the first layers of LSMO to present a 4-fold symmetry axis, perpendicular to the film, and untilted octahedra. It results, that in these layers, the crystal-field split $3 d$ orbitals of the $\mathrm{Mn}$ atom share their $(\vec{x}, \vec{y}, \vec{z})$ orthogonal axes with the $(\vec{a}, \vec{b}, \vec{c})$ substrate lattice vectors ( $\vec{c}$ being the out-of-plane direction); that is the Mn orbitals are $d_{a b}, d_{a c}, d_{b c}$ for the low energy ones and $d_{3 c^{2}-r^{2}}, d_{a^{2}-b^{2}}$ for the high energy ones. Any further distortion (such as Jahn-Teller) of the $\mathrm{MnO}_{6}$ octahedra should thus respect those constraints and are therefore restricted to atomic movements along the $\vec{c}$ direction. Let us now analyze the energy minimization of the LSMO layers at the interface under the above constraints. On one hand the film is under tensile strain and the minimization of the elastic energy favors a contraction of the LSMO monolayer u.c. along the $\vec{c}$ axis. On the other hand the film Fermi level orbitals are the Mn partially filled $d_{z^{2}}$ and $d_{x^{2}-y^{2}}$ ones while the lowest 
empty orbitals of the STO are the Ti empty $3 d$ orbitals. It is thus quite natural that the $e_{g}$ manganese orbitals delocalize to some extend into the titanium $3 d$ empty ones. This interaction is particularly favored in the case of the $3 d_{z^{2}}$ orbitals of the $\mathrm{Mn}$ and $\mathrm{Ti}$ atoms (see figure 1). Indeed, the through-bridge bonding mechanism

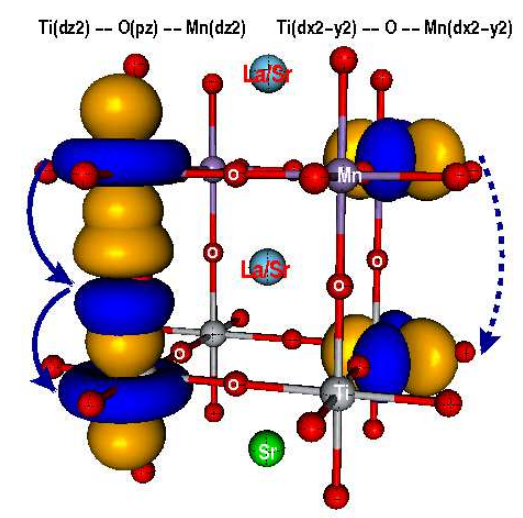

(b)

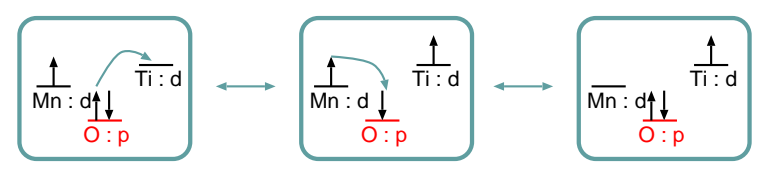

FIG. 1: (a) $d_{z^{2}}^{\mathrm{Mn}}-2 p_{z}^{\mathrm{O}}-d_{z^{2}}^{\mathrm{Ti}}$ and $d_{x^{2}-y^{2}}^{\mathrm{Mn}}-(\mathrm{O})-d_{x^{2}-y^{2}}^{\mathrm{Ti}}$ delocalization mechanism. (b) Through bridge delocalization mechanisms. The resulting effective Mn-Ti transfer integral is at the second order of perturbation

$t_{d^{2}, d_{z^{2}}^{\mathrm{Mn}}}=-\left\langle d_{z^{2}}^{\mathrm{Mn}}|\hat{H}| p_{z}^{\mathrm{O}}\right\rangle\left\langle\mathrm{O} p_{z}^{\mathrm{O}}|\hat{H}| d_{z^{2}}^{\mathrm{Ti}}\right\rangle /\left(\varepsilon_{d}-\varepsilon_{p}\right)$

acting via the $2 p_{z}^{\mathrm{O}}$ orbitals of the oxygens is energetically favored, due to the large $d_{z^{2}}^{\mathrm{Mn}}-2 p_{z}^{\mathrm{O}}-d_{z^{2}}^{\mathrm{Ti}}$ overlaps (fig. (1). This delocalization energy tends to favor a Jahn-Teller distortion increasing the occupation of the $d_{z^{2}}^{\mathrm{Mn}}$ orbitals, that is an elongation of the LSMO monolayer u.c. along the $\vec{c}$ axis. The physics at the interface thus results from the competition between

- the elastic energy that favors $c<a$,

$$
\frac{V}{2 \kappa}\left(\frac{\Delta V}{V}\right)^{2}
$$

- and the delocalization energy that favors $c>a$

$$
-\frac{\left(t_{d_{z^{2}}^{\mathrm{Mn}}, d_{z^{2}} \mathrm{Ti}}\right)^{2}}{\varepsilon_{d_{z^{\mathrm{T}}}^{\mathrm{Ti}}}-\varepsilon_{d_{z^{2}}^{\mathrm{Mn}}}}
$$

where $\quad t_{d_{z^{2}}^{\mathrm{Mn}}, d_{z^{2}}^{\mathrm{Ti}}} \simeq-\frac{\left\langle d_{z^{2}}^{\mathrm{Mn}} \mid p_{z}^{\mathrm{O}}\right\rangle\left\langle p_{z}^{\mathrm{O}} \mid d_{z^{2}}^{\mathrm{Ti}}\right\rangle}{\varepsilon_{d}-\varepsilon_{p}}$

On one hand, the relatively short metal-oxygen distances $(\simeq 1.95 \AA)$, the strong directionality of the $d_{z^{2}}$ and $p_{z}$ orbitals inducing a very large $\left\langle d_{z^{2}}^{\mathrm{Mn}} \mid p_{z}^{\mathrm{O}}\right\rangle\left\langle p_{z}^{\mathrm{O}} \mid d_{z^{2}}^{\mathrm{Ti}}\right\rangle$ overlap term, and the relatively weak orbital energy difference between the oxygen $2 p$ and the metal $3 d$ orbitals results in a very large effective transfer integral between the $d_{z^{2}}$ orbitals of the $\mathrm{Mn}$ and $\mathrm{Ti}$ atoms (of the order of $\simeq 1 \mathrm{eV} \underline{12}$ ) and thus of the delocalization energy. Indeed, one do not expect the $\varepsilon_{d_{z^{2}}^{\mathrm{Ti}}}-\varepsilon_{d_{z^{2}}^{\mathrm{Mn}}}$ energy difference to be larger than a few electron-Volts $(\sim 1,2)$. On the other hand, the elastic energy can be evaluated for different $c$ values. For a cubic structure $\left(c=c_{S T O}=3.905 \AA\right)$, the LSMO volume increase is of $2 \%$, and the associated elastic energy can be estimated to $\simeq 0.015 \mathrm{eV}$ (the LSMO compressibility being taken from ref. 13), that is much weaker (an order of magnitude) than the expected delocalization energy. Even if these energetic evaluations are only qualitative, one can expect with quite confidence, that for LSMO films (LCMO and similar films) on a STO substrate the first few ML at the interface are elongated in the $\vec{c}$ direction, despite their already in-plane tensile strain.

After a few unit cells, there is no more delocalization energy to gain from a larger occupation of the $d_{z^{2}}$ orbitals. At the same time, the in-plane symmetry operations imposed by the substrate should start to relax, and in particular the ones associated with the LSMO vibrational modes with the lowest frequencies : the octahedra tilt. The consequence of this constraint relaxation should be to adjust the $e_{g}$ orbitals occupations toward a bulklike value (compared to the first interface ML it means an increase of the $d_{x^{2}-y^{2}}$ occupation and a decrease of the $d_{z^{2}}$ one). The $c$ ML parameter should thus start to decrease toward the value expected from the elastic energy minimization (monolayer u.c. volume conservation), that is a contraction of the monolayers $c$ parameter and a tendency to a larger occupation of the $d_{x^{2}-y^{2}}$ orbitals compared to the $d_{z^{2}}$ ones.

What are the consequences of the above structural considerations, in term of magnetic and transport properties? As far as the first few layers at the interface are concerned, the tendency to occupy the $d_{z^{2}}$ orbitals at the expense of the $d_{x^{2}-y^{2}}$ ones should result in a decrease of the (in-plane) double-exchange and thus in a strong reduction of both the Curie temperature and the conductivity $\frac{19}{19}$. Let us note that even the $d_{z^{2}}$ orbitals are subject to the (in-plane) double-exchange, however the $d_{z^{2}}^{\mathrm{Mn}}-p_{x / y}^{\mathrm{O}}-d_{z^{2}}^{\mathrm{Mn}}$ delocalization process is much less effective than the $d_{x^{2}-y^{2}}^{\mathrm{Mn}}-p_{x / y}^{\mathrm{O}}-d_{x^{2}-y^{2}}^{\mathrm{Mn}}$ one, with the consequence that the effective (in-plane) exchange integral is much weaker and thus the Curie temperature. After a few layers, the relaxation of the $e_{g}$ orbitals occupation should result in an increase of the double exchange and thus of both the Curie temperature and the film conductivity. The temporary limit being set by the in-plane $a, b$ parameters constraints.

Finally, for very thick films this later constraint is relaxed and one should retrieve the bulk properties.

Let us now check the predictions issued from our model against the experimental data. LSMO thin films were grown by Laser-MBE using a setting detailed in ref. 17 . The temperature during the deposition is $620^{\circ} \mathrm{C}$ and the pressure is $4 \times 10^{-4}$ mbar. The gas is a mixture of $\mathrm{O}_{2}$ 
and $\mathrm{O}_{3}$. The intensity oscillations of the specular beam (RHEED) are used to measure the number of deposited layers. After the deposition, the pressure is increased to $5 \times 10^{-3}$ mbar associated with a higher $\mathrm{O}_{3}$ concentration, to insure a good oxidation. The films are then cooled to room temperature in this atmosphere. Such deposition conditions allow us to grow films with a $T_{c}$ higher than $325 \mathrm{~K}$ for thicknesses larger than $100 \AA$. After the growth, the films were studied by X-ray diffraction $(\theta-2 \theta$ mode) Seifert XP3000 system. From these data, the $c$ lattice parameter and the films thicknesses were determined. These measured thicknesses agree well with the number of RHEED oscillations. Magnetization measurements were carried out in a Quantum Design SQUID system, under a 500 Oersteds magnetic field parallel to the substrate. Transport measurements, four probes method, were carried out in a PPMS Quantum Design system.

LSMO ultrathin films with thicknesses ranging from $31 \AA$ (8 ML) to $92 \AA$ (24 ML) were grown onto 001oriented $\mathrm{SrTiO}_{3}$ substrates, previously etched to ensure a $\mathrm{TiO}_{2}$ terminating layer ${ }^{14}$. The out-of-plane average ML lattices parameters, $\langle c\rangle$, of the different films were determined by X-ray diffraction and are reported in table I] One sees immediately that the variation of $\langle c\rangle$ as a func-

\begin{tabular}{|c|c|c|c|}
\hline Thickness $(\AA)$ & Monolayers & $\langle c\rangle(\AA)$ & $\langle V\rangle\left(\AA^{3}\right)$ \\
\hline \multicolumn{2}{|c|}{ Expected values } & 3.823 & $58.29^{16}$ \\
\hline$>200$ & $>52$ & 3.847 & 58.66 \\
\hline 92 & 24 & 3.854 & 58.77 \\
\hline 70 & 18 & 3.856 & 58.80 \\
\hline 54 & 14 & 3.863 & 58.91 \\
\hline 31 & 8 & 3.874 & 59.07 \\
\hline
\end{tabular}

TABLE I: Film thicknesses $(\AA)$, numbers of LSMO monolayers, average monolayer $c$ parameters and average ML unit cell volume for LSMO films on STO (001) substrate.

tion of the number of ML does not correspond to the value expected from the conservation of the manganite volume. Indeed, the LSMO tensile strain at the interface let us expect a reduction of the ML average $\langle c\rangle$ parameter compared to the bulk value. Table $\amalg$ exhibits large $\langle c\rangle$ values associated with ML volumes larger than $V_{\text {bulk }}$. Such a behavior was also observed by Herger \& al15 on even thinner films with only 1,2,3,4,6 and 9 ML of LSMO. Our films show an increase of $\langle c\rangle$ when reducing the thickness (in agreement with films of ref. 15). Further examining the data of reference 15, one sees that while the first few layers at the interface exhibit $c$ values larger than the STO one (cumulative displacement compared to $c_{\mathrm{STO}}$ : $\Delta z$ positive and increasing), after about 3 monolayers $c$ retrieves a value smaller than $a=a_{\text {Sто }}$ (decreasing $\Delta z$ ). At this point, let us notice that all these structural results are in full agreement with our theoretical predictions.

Let us now examine the magnetic and transport properties. The magnetization of the different films is reported on figure2. For films thicker than $14 \mathrm{ML}$, the magnetization remains close to the values observed for the thickest films and the $T_{c}$ remains high. However, when the number of $\mathrm{ML}$ is decreased to 8, the magnetization at $10 \mathrm{~K}$ as well as $T_{c}$ are strongly decreased. These results agree well with the values reported in other works 7.9 for ultrathin LSMO films. The resistivity measurements

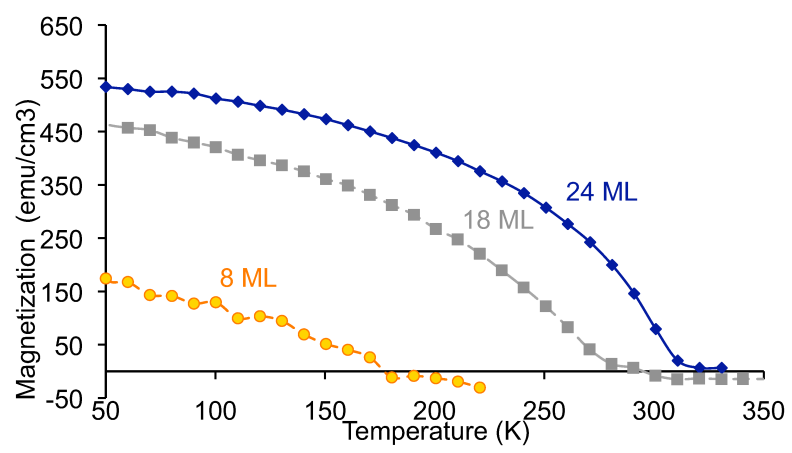

FIG. 2: Magnetization under a 500 Oersteds field of LSMO films on a STO (001) substrate.

agree with the suppression of the double exchange suggested for $8 \mathrm{ML}$ films on the magnetization curves (fig. 2). Indeed, the three thickest samples (14, 18 and $24 \mathrm{ML})$ exhibit a metal-insulator transition at $T_{c}$ while the thinner one $(8 \mathrm{ML})$ presents a semiconductor-like behavior with a large magneto-resistive effect. These peculiar magnetic and transport properties of the first few $(\sim 6-8)$ LSMO layers at the interface with STO are usually referred at as the dead layer ${ }^{7} \cdot \underline{9}$.

At this point let us notice that these experimental results do agree nicely with our theoretical predictions, and allow us to determine the number of layers over which each constraint extends. For instance the dominating $d_{z^{2}}$ occupation can be associated with only the first 2-3 ML (see $T_{c}$ or $\Delta z$ variation in the $9 \mathrm{ML}$ film of ref. 15 ). In the next layers the $e_{g}^{\mathrm{Mn}}$ orbitals occupations start to relax toward a more balanced one as supported by the increase of both $T_{c}$ and conductivity as a function of the number of layers in 3 to $8 \mathrm{ML}$ films. The fact that the $T_{c}$ value reaches its saturation limit, that the metalinsulator transition is restored tells us that after about 8 $\mathrm{ML}$, the double-exchange is fully restored and thus the manganese $e_{g}$ orbitals occupations.

The last point of our predictions one would like to see verified, is the actual dominating $d_{z^{2}}^{\mathrm{Mn}}$ occupation in the first layers at the STO interface. LD-XAS experiments were conducted by different authors that reached opposite conclusions. Indeed, while Tebano et $a \underline{\underline{\eta}}$ report the signature of a preferential $d_{z^{2}}$ orbital occupation at the interface, Huijben et al argue that the experimental evidences are not significant. Very recently ARPES measurements ${ }^{8}$ were done on 4,6 and $10 \mathrm{ML}$ films and clearly exhibit an increase of the $\Gamma$ point signal (attributed to the $d_{z^{2}}^{\mathrm{Mn}}$ orbitals contribution) when the thickness of the film decreases. It thus seem that the experimental data confirm our findings from constraints and energetic considerations.

Another way to check the validity of our interface 
model is to test it against the properties of ultrathin films deposited on a buffered substrate. Indeed, the deposition of a buffer layer at the interface between the STO and the LSMO should strongly modify the electronic structure of the first LSMO layers. We thus grew either a $\left(\mathrm{LaMnO}_{3}\right)\left(\mathrm{SrMnO}_{3}\right)$ buffer (BUF1) or a $\left[\left(\mathrm{LaMnO}_{3}\right)\left(\mathrm{SrMnO}_{3}\right)\right]_{2}(\mathrm{BUF} 2)$ on STO, prior to the LSMO film. Let us point out that the $\left(\mathrm{LaMnO}_{3}\right)\left(\mathrm{SrMnO}_{3}\right)$ superlattices present an antiferromagnetic behavior ${ }^{18}$, when deposited in these conditions. The buffer layer will thus break both the symmetry constraint preventing the LSMO octahedra to be tilted in the first LSMO layers at the interface and the possibility for the LSMO manganese to delocalize its $d_{z^{2}}$ population in the empty $d_{z^{2}}^{\mathrm{Ti}}$ orbitals. Of course some delocalization of the LSMO $d_{z^{2}}^{\mathrm{Mn}}$ orbitals can still occur since the buffers $d_{z^{2}}^{\mathrm{Mn}}$ orbitals are most probably not totally filled. This effect is however expected to be much weaker than at the LSMO/STO interface both because of the buffer $d_{z^{2}}^{\mathrm{Mn}}$ orbital occupation and because of the starting of the $\mathrm{MnO}_{6}$ octahedra tilt in the LSMO. We thus expect that the buffered LSMO films will be more "bulklike" than the unbuffered ones, the thicker the buffer, the more "bulk-like" the film (the wider the distance between the film and the STO surface, the lesser the symmetry constraints will be imposed to the LSMO). It means that for equal film thicknesses, the thicker the buffer, the highest the $T_{c}$ and the film conductivity should be.

\begin{tabular}{cc}
\hline Substrate & $\langle c\rangle(\AA)$ \\
\hline Bare & 3.874 \\
Buffered $($ LMO) $($ SMO $) \times 1$ & 3.871 \\
Buffered $($ LMO $)($ SMO) $\times 2$ & 3.862 \\
\hline
\end{tabular}

TABLE II: Average $\langle c\rangle$ ML parameters for 8 ML LSMO films deposited either on bare or on buffered substrates.

Table II experimental data show that the average $\langle c\rangle$ ML parameter decreases when the number of buffer layers goes from 0 to 2 . This results in a decrease of the $c / a$ ratio and induces a larger occupation of the $d_{x^{2}-y^{2}}$ orbitals with respect to the $d_{z^{2}}$ ones. Such an electronic structure favors a more robust ferromagnetic behavior of the films as the thickness of the buffer layer increases. Indeed, both the magnetization (see fig. 3) and $T_{c}$ increase with increasing buffer thickness, as expected from the $c / a$ ratio. The film buffered by two (LMO)(SMO) layers exhibits a $T_{c}$ close to $275 \mathrm{~K}$, that is about $100 \mathrm{~K}$ higher than the $T_{c}$ observed for the film of same thickness deposited on a bare STO substrate. The resistivity of the buffered films behave as for a regular ferromagnetic materials with a maximum value at $T_{c}$.

In conclusion, we presented in this paper a simple model able to explain and predict the interface effects between perovskites oxides. We applied our model to the difficult case of the nature and origin of the so-called dead layer at the LSMO interface with an STO substrate. Our model successfully predicted and explained all experimental features of this interface, up to now not under-

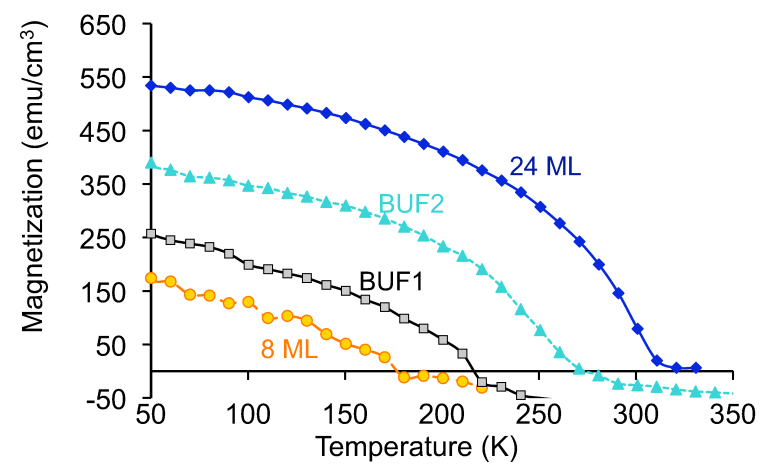

FIG. 3: Magnetization under a 500 Oersteds field, of 8 ML LSMO films deposited on bare or buffered (BUF1: $(\mathrm{LMO})(\mathrm{SMO}) \times 1 ; \mathrm{BUF} 2:(\mathrm{LMO})(\mathrm{SMO}) \times 2) \mathrm{SrTiO}_{3}$ substrates. The magnetization of an unbuffered $24 \mathrm{ML}$ film is given for comparison.

stood.

\section{Acknowledgments}

The authors thank ANR program SEMOME and STREP MACOMUFI for the support of this work.
1 A. Sadoc, B. Mercey, Ch. Simon, D. Grebille, W. Prellier, M.-B. Lepetit, Phys. Rev. Letters. 104, 046804 (2010).

${ }^{2}$ F. Tsuia, M. C. Smoak, T. K. Nath and C. B. Eom, Appl. Phys. Letters 76, 2421 (2000).

3 C. Adamo, X. Ke, H. Q. Wang, H. L. Xin, T. Heeg, M. E. Hawley, W. Zander, J. Schubert, P. Schiffer, D. A. Muller, L. Maritato, and D. G. Schlom1, Appl. Phys. Letters 95, 112504 (2009).

${ }^{4}$ M. Ziese, H. C. Semmelhack and K. H. Han, Phys. Rev. B 68, 134444 (2003).

5 I. C. Infante, F. Sánchez, J. Fontcuberta, M. Wojcik, E. Jedryka, S. Estradé, F. Peiró, J. Arbiol, V. Laukhin and J. P. Espinós, Phys. Rev. B 76, 224415 (2007).
${ }^{6}$ M. Bibes, Ll. Balcells, S. Valencia, J. Fontcuberta, M. Wojcik, E. Jedryka, and S. Nadolski, Phys. Rev. Letters 87, 067210 (2001).

7 A. Tebano, C. Aruta, S. Sanna, P. G. Medaglia, G. Balestrino, A. A. Sidorenko, R. DE Renzi, G. Ghiringhelli, L. Braicovich, V. Bisogni, N. B. Brookes, Phys. Rev. Letters 100, 137401 (2008).

8 A. Tebano, A. Orsini, P. G. Medaglia, D. Di Castro, G. Balestrino, B. Freelon, A. Bostwick, Young Jun Chang, G. Gaines, E. Rotenberg and N. L. Saini, Phys. Rev. B 82, 214407 (2010).

9 M. Huijben, L. W. Martin, Y.-H. Chu, M. B. Holcomb, P. Yu, G. Rijnders, D. H. A. Blank, R. Ramesh, Phys. Rev B 
78, 094413 (2008).

10 H. Yamada, Y. Ogawa, Y.H. Sato, M. Kawasaki, H. Akoh, Y. Tokura, Science, 305, 646 (2004).

11 M. V. Abrashev, A. P. Litvinchuk, M. N. Iliev, R. L. Meng, V. N. Popov V. G. Ivanov, R. A. Chakalov and C. Thomsen, Phys. Rev. B 59, 4146 (1999).

12 R. Bastardis, N. Guihry, N. Suaud and C de Graaf, J. Chem. Phys. 125, 194708 (2006).

13 L. Glasser, J. Phys. Chem. C 114, 11248 (2010) ; and references therein.

14 M. Kawasaki, K. Takahashi, T. Maeda, R. Tsuchiya, M. Shinohara, O. Ishiyama, T. Yonezawa, M. Yoshimoto, H. Koinuma, Science,226, 1540, (1994)
15 R. Herger, P. R. Willmott, C. M. Schlepüz, M. Bjöck, S. A. Pauli, D. Martoccia, B. D. Patterson, D. Kumah, R. Clarke, Y. Yacoby, M. Döbeli, Phys. Rev. B 77, 085401 (2008).

16 G. Venkataiah, V. Prasad and P. Venugopal Reddy, Journal of Alloys and Compounds 429, 1 (2007).

17 B. Mercey, P. A. Salvador, Ph. Lecoeur, W. Prellier, M. Hervieu, Ch. Simon, D. Chippaux, A. M. Haghiri-Gosnet, B. Raveau, J. of Appl. Phys. 94, 2716 (2003).

18 J. Verbeeck, O. I. Lebedev, G. Van Tendeloo and B. Mercey, Phys. Rev. B 66, 184426 (2002).

19 P.-G. De Gennes, Phys. Rev. 118, 141 (1960). 